\section{LR White Flat Embedding Of Cells Grown On Coverslips}

Tamara Howard, Cold Spring Harbor Laboratory howard@cshl.org

We do this two different ways, using polyethylene (PE) molds or aluminum weigh boats, depending on the sample. You can either heat- or UV-cure these molds - just change the type of "lid" on the embedding mold.

The PE molds are used when the coverslip can be made to fit in the mold. We use JB-4-type molds, available from your favorite microscopy supplier. The key to using these molds is that they have to be pre-treated...fill the inner cup of the mold with LR White, cover with an aluminum JB-4 chuck (try to have enough resin in the mold to come up around the base of the chuck), and polymerize in a $60^{\circ} \mathrm{C}$ oven for a day or two, until the resin is hard. The Al chuck can usually be removed by hand, but a flathead screwdriver used as a pry will help pop it off if you have trouble. Dispose of the resin block and wipe out the cup with KimWipes...the mold is ready to use for real samples. The Al chuck can be cleaned by soaking it for a day in methanol (in the hood). Any resin remaining stuck to the chuck can be scraped off with a spatula. Our guess is that there are micropores in the molds that have to be sealed; we've had problems with incomplete polymerization of blocks when fresh molds were used. The molds last until you get sick of them or until someone turns up the oven without checking to make sure it is empty and melts everything. Thermanox or other plastic coverslips can be cut to fit the molds (before the cells are plated!); glass coverslips can be broken to fit. Tissue pieces that are too large for embedding capsules or that have to be oriented can also go in these molds.

The Al dishes are for samples that can't be made to fit into the PE molds. I use these for gridded coverslips when we do correlative microscopy of single cells. Gridded coverslips always seem to break "funny", leaving you with shards of glass. So, I leave them whole and embed in the Al boats. They require more resin, but I think it is worth it in terms of ease of use.

The fixation and dehydration are done in culture dishes. The coverslips are transferred to glass containers (dishes, scintillation vials, whatever) for the resin infiltration steps, since LR White eats polystyrene. Or you can infiltrate in Al boats, but the resin use increases drastically.

When you are ready to embed, put a few drops of resin in the base of the mold, then put in the coverslip, cells up. (We clip the upper right corner of Thermanox coverslips; for glass, you can ₹ usually see which side the cells are on, or check in a dissecting $\frac{\oint}{3}$ 'scope.) Then fill the PE mold's cup with resin. With Al boats, fill o halfway with resin. If you are going to heat-cure, use $\mathrm{Al} \mathrm{JB}-4 \stackrel{\circ}{\circ}$ chucks as lids for the PE molds, or another Al boat for the Al boat molds. Make sure you have resin at least up to the lower surface of the lid, to ensure an air-free polymerization. This is tricky with the Al boats - I try to add enough resin to come close to the top of the lower (mold) boat. The resin will shrink a bit during polymerization, but there is enough depth in these molds to keep the sample in resin if you've put enough resin in to start with! Use regular lab tape to label the samples; it will stick to the Al of the chuck or boat.

To UV-cure, use Aclar or heavy Saran-type wrap to cover the top of the mold/boat. It will sit on the resin surface; try to avoid forming air pockets.

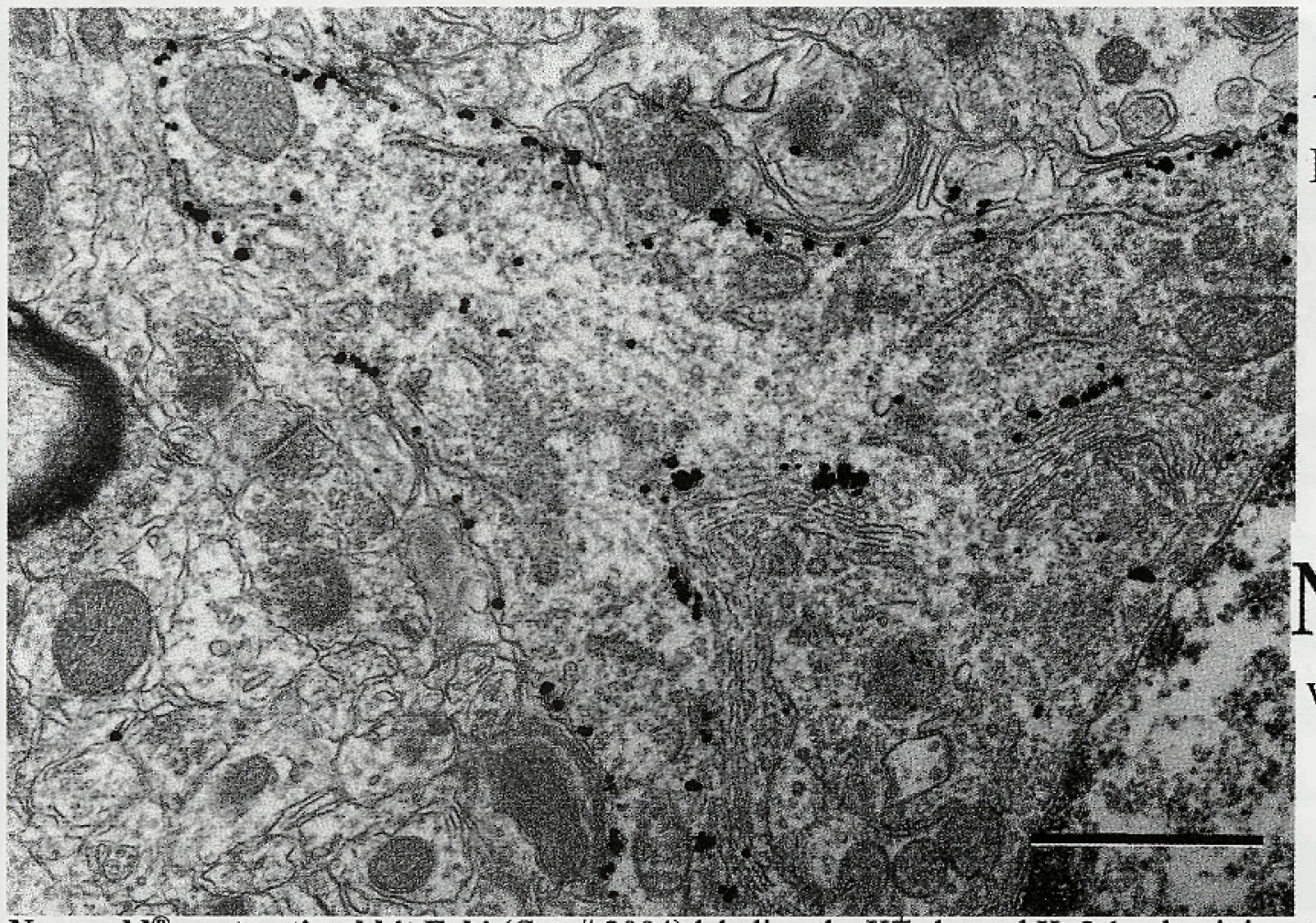

\title{
Nanogold!
}

\section{Better:}

*Labeling Density *Specificity *Resolution

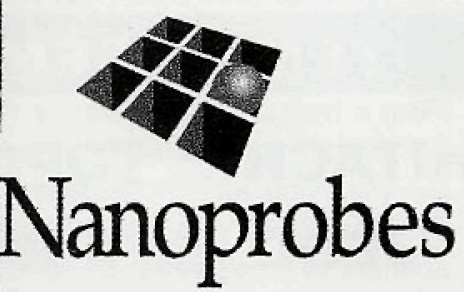
www.nanoprobes.com

$877-447-6266$

(US Toll-Free)

631-205-9490

Fax: 631-205-9493

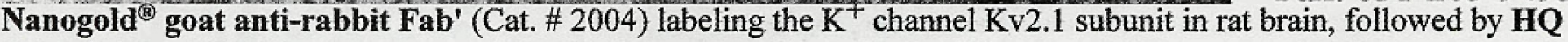
Silver $^{\text {(8) }}$ (Cat. \# 2012) enhancement. Note high density and specificity of immunostaining, even elucidating subunit localization to cytoplasmic side of cell membrane and outer stacks of the Golgi; axons and terminals clearly negative. Bar $=1$

$\mu \mathrm{m}$. Reprinted from Neuroscience, 84, J. Du, J.-H. Tao-Cheng, P. Zerfas, and C.J. McBain. The K ${ }^{+}$channel Kv2.1 is apposed to astrocytic processes and is associated with inhibitory postsynaptic membranes in hippocampal and cortical principal neurons and inhibitory interneurons, 37-48, Copyright 1998, with permission from Elsevier Science. 